\title{
Histopathological evaluation and molecular detection of natural iridovirus infection in cultured grouper fish in Malaysia
}

\begin{abstract}
Iridovirus is the causative agent of grouper iridovirus infection (GIV), which causes severe epizootics resulting in large-scale mortalities and huge economic losses in cultured and marine fishes worldwide. The current study evaluated the gross and histopathological lesions, and molecular detection of GIV in naturally infected grouper farms in Malaysia. A total of 150 moribund fish showing different clinical signs, presented to the Aquatic unit were used for this study. The fish were necropsied and visceral organs (spleen, kidney, heart, liver, intestine, brain, eyes, gills, and some parts of skin with ulcer) were collected and fixed in 10 $\%$ buffered formalin for histopathological processing and evaluation. Molecular detection of the virus was done by polymerase chain reaction (PCR). Grossly, the groupers had ulceration on the operculum and close to the caudal fin with hemorrhages on the margin of the caudal fin. In some fish, there was necrosis of the caudal fin, sloughing of the epidermis, dermal ulceration and popeyes Histological sections showed large basophilic cytoplasmic inclusions and vacuolations in the cytoplasm of hepatocytes. Basophilic or eosinophilic enlarged cells with the presence of mononuclear cellular infiltrations were seen in the kidney, liver, eye and gills, which is the distinctive feature of this disease. PCR detection showed positive amplification from 27 groupers. Based on the gross, histopathological and molecular detection GIV infection was established.
\end{abstract}

Keyword: Grouper; Histopathology; PCR; Iridovirus; GIV 